\section{Appréciation du TARMED}

Chères consœurs,

Chers confrères

La Fédération des médecins suisses (FMH) organise pour la deuxième fois de son histoire une votation générale auprès de ses membres, à propos de l'introduction des tarifs TARMED.

Comme vous le savez sans doute, la FMH a développé ces tarifs avec les assureurs, à savoir la CTM (Commission des tarifs médicaux des assureurs fédéraux dans le domaine de l'assurance-accidents [AA]), l'Assurance-invalidité (AI) et l'Assurance militaire (AM), dans le cadre du projet GRAT, puis du projet TARMED (avec également santésuisse [anciennement CAMS]) et $\mathrm{H}+$ en qualité d'organisation des hôpitaux). Après onze ans de travaux, ces tarifs sont prêts à être introduits.

\section{Considérations générales}

Rappelons une fois de plus les points essentiels du projet:

\section{Nomenclature tarifaire unifiée}

Dans le domaine de l'assurance-accidents (LAA), comme dans celui de l'assurance-maladie (LAMal), une seule nomenclature des prestations médicales (désignation avec interprétations éventuelles, points tarifaires) sera utilisée. Pour la LAMal, cela représente une nouveauté fondamentale qui est, cela dit, prescrite par la loi (art. 43, $5^{\mathrm{e}}$ al., LAMal). liées:

Un certain nombre d'implications de base y sont

- Dans le domaine de la LAMal également, les prestations médicales peuvent être comparées en fonction des coûts générés, de l'endroit où la prestation est apportée et d'autres caractéristiques.

- Toutes les structures tarifaires utilisées jusqu'ici sont caduques et doivent être remplacées par la structure tarifaire TARMED à laquelle le Conseil fédéral a donné son aval. En conséquence, toutes les anciennes conventions tarifaires ont été dénoncées. Il est dès lors illusoire de penser pouvoir rejeter la structure tarifaire TARMED et continuer d'appliquer les anciens tarifs ou une autre structure tarifaire.

- Les nombres de points tarifaires ne donnent la somme remboursée au médecin qu'en les multipliant par une valeur du point exprimée en francs. Dans le domaine LAA, la valeur du point tarifaire
(VPT) pour toute la Suisse a été fixée avec les assureurs-accidents à Fr. 1.-. Dans le domaine de la LAMal, au moins 26 VPT (une par canton) devront, par principe, être appliquées. Les négociations avec santésuisse relèvent des sociétés cantonales de médecine. Elles ne pourront commencer que lorsqu'un consensus aura été trouvé sur la structure tarifaire ou plutôt sur les dispositions d'introduction et consigné dans une conventioncadre à l'échelle confédérale. L'exigence souvent avancée selon laquelle il ne faudra voter sur le TARMED que lorsque les VPT cantonales auront été fixées est donc irréalisable.

\section{Application des tarifs TARMED}

Les tarifs TARMED ne pourront être appliqués que dans le domaine ambulatoire des soins en cabinet médical ou en section ambulatoire de l'hôpital, mais pas dans le domaine hospitalier. Ce point n'était pas clair lors de la phase initiale du projet GRAT/TARMED. C'est pourquoi on a établi, à l'époque, une nomenclature complète et une tarification de toutes les prestations médicales (donc ambulatoires et hospitalières). Même si la facturation des prestations médicales hospitalières ne se fera pas directement par les tarifs TARMED, ce procédé s'est révélé utile, car

- la démarcation entre prestations ambulatoires et hospitalières n'est pas fixe et

- le transfert ou l'utilisation dans d'autres systèmes tarifaires (groupes homogènes de malades [DRG], autres modes de rémunération forfaitaires) est ainsi possible.

\section{Autres tarifs médicaux}

Hormis les tarifs médicaux proprement dits, il en existe un grand nombre d'autres qui contiennent des prestations également apportées par des médecins. Citons p. ex. le tarif de physiothérapie, le tarif des dentistes et surtout la liste des analyses qui peut être qualifiée de structure tarifaire fédérale pour les analyses de laboratoire et remplacera les tarifs de laboratoire cantonaux (pour autant que ce ne soit déjà le cas). Les prestations de ces tarifs ayant une importance pour les médecins n'ont pas été reprises dans les tarifs TARMED.

Pour l'application des tarifs non médicaux exposés plus haut, ce qui suit est valable:

- Les tarifs sont appliqués également par des médecins selon la convention tarifaire entre fournisseurs de prestations et assureurs. Cela comprend également les éventuelles sanctions (exception: liste des analyses).

- La liste des analyses sur l'ensemble du territoire est introduite avec les conventions-cadres correspondantes et avec une VPT fixée par le Département fédéral de l'intérieur (DFI). Les sanctions éventuelles sont infligées selon les conventionscadres. Les activités de QUALAB dans ce domaine prendront fin avec l'introduction des conventions-cadres. 


\section{Champ d'application}

Les tarifs TARMED ne trouveront leur emploi formel que dans le champ d'application de l'assurance-maladie sociale et de l'assurance-accidents, invalidité et militaire, mais pas dans le domaine de l'assurance complémentaire. Soumis à la pression de la Commission de la concurrence (COMCO), ce domaine en constante régression, alors que le nombre de médecins ne cesse de croître, est de plus en plus en butte au libre marché et à la pression des prix; les réglementations tarifaires ont été abrogées. Il serait naïf cependant de vouloir nier aux tarifs TARMED le rôle de référentiel. Ces préliminaires expliquent l'opposition des médecins en chirurgie opératoire et interventionnelle.

\section{Diminution du revenu?}

- Une comparaison des prix des prestations ambulatoires souvent apportées (selon les tarifs actuels de l'assurance sociale) avec les tarifs TARMED ne montre pas, après pondération des volumes et avec une VPT de Fr. 1.-, de diminution significative $(<10 \%)$ des revenus si les nouveaux tarifs sont correctement appliqués et si l'attention n'est pas fixée sur le prix de la prestation au sens strict du terme. La seule exception concerne les radiologues pour lesquels il faut prendre des mesures d'ici à l'introduction des tarifs afin d'assurer leur existence. Si cela se révélait possible, le Comité central bloquerait l'introduction des tarifs même en cas d'acceptation en votation générale.

- Les comparaisons des prix et des revenus seront rendues plus difficiles par le fait que des particularités qui se sont glissées lors de l'application de la plupart des tarifs ont fait naître une autre réalité tarifaire sous le couvert des tarifs officiels. C'est le cas de la plupart des tarifs, qu'il s'agisse du catalogue des prestations hospitalières ou des tarifs cantonaux LAMal. Seuls les calculs de référence de NewIndex, fondés sur des réalités de facturation conformes à la réalité matérielle et transcodés de manière appropriée, donnent des résultats utilisables. Les comparaisons se fondant exclusivement sur l'application à la lettre des tarifs actuels sont dès lors, quel que soit leur instigateur, insensés et trompeurs.

\section{Données/protection des données}

Le but visé par les assureurs-maladie était - et est encore - de profiter de l'introduction des tarifs TARMED pour acquérir des données aussi détaillées que possible sur les patients et, encore plus, sur les fournisseurs de prestations. Il s'agit là d'un domaine extraordinairement sensible tant sur les plans éthique et juridique que politique. Des concessions susceptibles de porter atteinte aux principes fondamentaux de notre politique sont impensables dans ce domaine.

- Ce sont les dispositions en matière de protection des données qui tout d'abord imposent ici des limites. Malheureusement, il n'a pas été possible d'examiner à fond ces questions avec les fonctionnaires de la protection des données.
- Les assureurs-maladie exigent qu'on leur apporte sur un plateau toutes les banques de données de la FMH concernant ses membres. Concrètement, cela signifierait que toutes les données ayant trait à nos membres devraient être fournies régulièrement aux assureurs (c.-à-d. à chaque caisse-maladie et à ses agences) sur support électronique, et ce sans la moindre garantie crédible de respect de la confidentialité. Cette attitude des assureursmaladie est tout à fait compréhensible de leur point de vue: ils veulent entrer en possession des données pour ainsi dire "avant la lettre», afin de pouvoir, après la suppression de l'obligation de contracter, sélectionner les médecins qui leur conviennent. Comme il n'est manifestement pas si facile de réunir soi-même ce genre de données et de les gérer - pensons à SWICA -, il est bien plus simple de se les procurer par contrat et, qui plus est, gratuitement auprès de celui qui dispose de la meilleure banque de données sur le plan qualitatif.

Cette façon de faire est bien entendu impensable et c'est pourquoi il n'y a pas d'annexe à ce propos dans la convention-cadre avec les assureurs-maladie ni dans les documents soumis à la votation. Si une telle réglementation devait être convenue avec santésuisse, elle serait alors soumise à la décision de la Chambre médicale. Avant une décision de la Chambre médicale, le Comité central bloquerait l'introduction des tarifs TARMED, même en cas de résultat positif de la votation générale.

Les assureurs doivent enfin comprendre que la protection des données et celle de la personnalité ne sont pas négociables et que des menaces dignes de l'âge de la pierre n'y changeront rien.

\section{Concept de valeur intrinsèque}

Le concept sur la valeur intrinsèque et celui concernant les unités fonctionnelles sont des outils indispensables pour le maniement de la structure tarifaire et ne peuvent donc en être dissociés. Ils sont par conséquent soumis à la décision du corps médical avec la structure tarifaire, dans le cadre de la votation générale, sous la forme approuvée par la direction générale du projet TARMED, à savoir la version 9.0 (pour le premier) et la version du 28 août 2001 (pour le second). Ces concepts ne font cependant pas partie de la structure tarifaire au sens de l'art. 43, $5^{\mathrm{e}}$ al., LAMal et ne sont soumis à aucune intervention de la part des autorités.

En résumé, le concept sur la valeur intrinsèque fixe les prestations facturables par les médecins en fonction de leur formation postgraduée; dans la structure tarifaire, cette indication apparaît sous la rubrique valeur intrinsèque qualitative. Quant à la valeur intrinsèque quantitative, elle n'a en fait pas d'importance pour les utilisateurs de la structure tarifaire TARMED; elle indique simplement selon quelle valeur intrinsèque une prestation a été calculée. Ces données peuvent être consultées, pour chaque prestation, dans le Closed-User-Group (CUG) créé à cet 
effet sous www.tarmed.ch; elles n'apparaissent pas dans le navigateur tarifaire, car la différence entre valeur intrinsèque quantitative et qualitative est manifestement difficile à comprendre et peut être source de malentendus.

\section{Remaniement ("reengineering»)}

Avant son introduction, la version 1.1 de la structure tarifaire TARMED devait encore subir une vérification de principe des valeurs-clés et des variables de pilotage, des relations entre ces valeurs (ou de l'algorithme de calcul), ainsi que des données de base. Ce processus désigné sous le nom de remaniement II (RE II) devra être terminé avant l'introduction dans le domaine LAMal, c.-à-d. avant le 31 décembre 2002. Si cet objectif devait ne pas être atteint dans le délai, le Comité central bloquerait alors l'introduction des tarifs TARMED.

La remarque de principe suivante s'impose dès lors: une structure tarifaire ne peut jamais être "définitive", à moins d'un arrêt complet des progrès de la médecine. C'est pourquoi un accord donné à un certain moment ne peut porter que sur ce qui a été atteint à ce moment précis.

Bien que, tant pour des raisons financières qu'opérationnelles, il eût été préférable d'effectuer la votation générale et le recensement des valeurs intrinsèques en même temps, le CC a renoncé à procéder de cette manière, même après consultation de la Chambre médicale. L'important était en effet d'éviter tout impression d'une tentative d'influencer la votation générale en cours.

\section{Critiques actuelles}

1. TARMED contient encore un grand nombre d'erreurs et n'est pas acceptable sous cette forme

- Il faut reconnaître tout d'abord que les travaux de mise à jour qui ont abouti à la version 1.1 ont donné lieu à d'importantes fautes matérielles. [changements non conformes aux décisions entre la version 1.0 et la 1.1; psychiatrie: erreurs dans la prise en compte du facteur 1,2; psychothérapie déléguée: pas seulement pour les spécialistes en psychiatrie; norme XML: ne doit pas être ancrée dans la structure tarifaire].

- Des reproches de ce genre émanent en bonne partie de la Suisse romande; en règle générale, ceux-ci proviennent de problèmes survenus dans la traduction d'allemand en français.

- Mais très souvent, on qualifie d'erreur le fait qu'une exigence médicale n'a pas pu être concrétisée. L'explication sous-jacente en est une fausse représentation des négociations tarifaires. En effet, les délégations de médecins sont loin de pouvoir faire accepter leurs exigences comme une ordonnance médicale; la réalité est bien différente: les assureurs contestent en principe toutes les exigences des médecins ou leur opposent des contre-exigences.
On peut se plaindre d'une telle forme de négociations comme étant fausse et inappropriée; mais, en Suisse (comme dans la plupart des autres pays), elle est garantie par la loi; c'est même une réalité imposée.

2. Le TARMED comprend plus de 2000 prestations mettant en danger les patients et menaçant la qualité des soins médicaux

Ces critiques sont inqualifiables et démagogiques:

- Cet argument est avancé maintenant alors que rien de fondamental n'a été changé à la nomenclature depuis la version 2.2. Sans compter qu'il est le fait de représentants de sociétés de discipline médicale qui - les preuves existent - ont collaboré depuis le début et jusqu'à maintenant à l'élaboration des structures tarifaires sans avancer la moindre objection à cet égard.

- En comparant le rapport prix-prestation sur un plan international, on constate que les tarifs TARMED viennent en tête de classement. Pourtant, dans les pays présentant un rapport prixprestation parfois bien plus bas, on n'a pas pu établir d'augmentations de morbidité et de mortalité induites par le tarif.

- Les tenants de cette critique n'ont jusqu'ici pas pu nommer une seule prestation qui confirmerait en substance ce soupçon effroyable. A la Chambre médicale, trois exemples ont été cités, qui n'en apprennent pas plus sur les menaces insinuées mais qui en disent long sur les lacunes de compréhension tarifaire des auteurs concernés.

\section{L'introduction du TARMED coûtera à chaque} cabinet médical des milliers de francs d'équipement et de logiciels de facturation

C'est là un mythe propagé par les fournisseurs de logiciels qui entendent profiter de l'aubaine pour prendre une petite (voire une grande) part de gâteau ou transformer en espèces sonnantes et trébuchantes les mises à jour programmées à long terme dans le futur.

En réalité, il s'agit de développer un système qui - est adapté au TARMED et peut effectuer des calculs avec des points tarifaires PM et PT dotés de différentes valeurs;

- permet au médecin de facturer les prestations correspondant à sa valeur intrinsèque qualitative;

- comprend les médicaments, pansements, etc. (comme jusqu'ici) et le tarif de physiothérapie, celui des dentistes et la liste des analyses.

Ces précisions sont connues de toutes les maisons de logiciels depuis plus d'une année (et sont également accessibles dans le CUG TARMED). Le problème se trouve donc ailleurs:

- Beaucoup de ces firmes n'ont pas voulu investir tant qu'une décision définitive n'avait pas été prise sur l'introduction du TARMED.

- Elles ont maintenant un problème pour équiper tous leurs clients jusqu'au délai d'introduction, fixé au $1^{\mathrm{er}}$ janvier 2003 au plus tard. Les explica- 
tions ci-dessus montrent cependant que les coûts impliqués, pour peu qu'ils concernent le travail lui-même, ne sauraient s'élever à des milliers de francs.

4. Une décision sur le TARMED n'est pas possible tant qu'on ne connaîtra pas toutes les valeurs cantonales du point tarifaire

Cette position est tout à fait compréhensible à première vue; personne n'achète en effet des bananes selon un prix indiqué en nombre de points tarifaires qui, à la caisse sera multiplié par une valeur du point inconnue pour obtenir le prix définitif. Mais c'est une fois de plus la logique légale, peu accessible au «sens commun", qui l'exige:

- Pour le domaine AA/AM/AI, c'est possible, car il s'agit d'un tarif fédéral pour lequel UNE SEULE VALEUR DU POINT est négociée entre les parties à la convention, à savoir la FMH et les assureurs fédéraux sociaux. La valeur adoptée à l'issue de cette négociation - comme on peut le voir dans les documents - est de Fr. 1.-, sous réserve de l'approbation du Conseil fédéral prescrite par la loi.

- La situation est fondamentalement différente dans le domaine de la LAMal, où la valeur du point doit être négociée dans 26 cantons, recevoir la bénédiction du surveillant des prix et être approuvée par le gouvernement cantonal, sur la base d'une structure tarifaire fixée au niveau fédéral.

- De cette configuration, il ressort clairement que les négociations cantonales sur la valeur du point ne pourront commencer que lorsque la structure tarifaire aura été fixée au niveau fédéral. De plus, au vu de ce qui précède, la demande visant à procéder en même temps à un vote sur la structure tarifaire ET sur la valeur du point cantonale est irréalisable tant pour des raisons de technique de négociations que pour des raisons légales.

\section{Perspectives}

Au cours des dernières semaines, l'impression est apparue au sein de la population, mais aussi parmi les médecins, que la votation générale à venir allait décider du sort des tarifs TARMED. Or, ce n'est pas le cas, notamment si le résultat est un OUI:

- La FMH ayant jusqu'ici (contrairement à ce que certains espéraient en son sein) refusé de condamner le TARMED, d'autres partenaires semblent être désormais dans l'embarras: santésuisse n'a pour l'instant pas encore donné son aval à la convention-cadre et la dernière prise de position de $\mathrm{H}+$ soulève plus de questions qu'elle n'apporte de réponses.

- Toutes les décisions des parties contractuelles, comme celles des autorités compétentes, sont susceptibles de recours. On peut s'attendre, avec certitude, à ce qu'il soit fait usage de cette possibilité, d'autant plus que la voie de droit prend du temps et qu'elle permet donc d'en gagner (cf. Zemp Gsponer C, Bitzi F. TarMed und Art. 6
EMRK. Besteht ein Anspruch auf gerichtliche Überprüfung nach der Europäischen Menschenrechtskonvention? Bull Méd Suisses 2001;82[18]: 924-7). Du côté des autorités compétentes, on minimise et on banalise le problème; aucune mesure concrète n'a d'ailleurs été prise.

Cela dit, la FMH ne peut ni ne doit en tirer prétexte pour repousser ses décisions; bien au contraire. En rapport avec celles-ci, une question revient constamment: celle des conséquences d'un rejet des tarifs TARMED par la FMH. Tout d'abord, il est noter que, dans aucun pays du monde (et les exemples ne manquent pas), un tarif de prestations médicales n'a pu être introduit contre la volonté déclarée du corps médical. Et il en serait de même en Suisse dans le cas où les autorités politiques introduiraient la version 1.1 du TARMED, par le biais d'un arrêté fédéral urgent (AFU) par exemple, et en fixeraient l'application (notamment la valeur du point) dans une ordonnance.

Les conséquences d'une telle démarche seraient prévisibles:

- Le corps médical ou, plus précisément, la FMH refuserait de coopérer dans l'application des tarifs et de se plier aux dispositions tarifaires. Le tarif serait ainsi en grande partie inutilisable.

- Entre les assureurs et les instances politiques, d'une part, et le corps médical, d'autre part, un état de guerre chronique régnerait. Les médecins feraient quelque chose qu'ils n'ont jamais fait jusqu'ici: ils feraient corps et lanceraient un assaut frontal contre ces institutions. Ce ne serait certainement pas une partie de plaisir pour ces institutions qui, aujourd'hui déjà, doivent se débattre avec d'importants problèmes de crédibilité et d'image.

Mais à cela s'ajouteraient d'autres conséquences négatives, à long terme notamment:

- Les médias, comme les milieux politiques et les assureurs, auraient une fois de plus l'occasion de condamner les médecins comme les éternels empêcheurs de tourner en rond, responsables de tous les maux du système de santé et de détourner ainsi l'attention de leur propre incompétence et de leur incapacité en qualité de concepteurs.

- Le plaisir de nombreux politiciens, notamment, à asservir ce corps médical par tous les moyens légaux et juridiques ne ferait qu'augmenter, empêchant l'instauration d'un environnement professionnel satisfaisant régi par le principe d'autonomie de l'activité médicale.

- Le corps médical serait relégué au second plan; il ne pourrait plus contribuer de manière constructive aux prises de décision.

Pour l'essentiel, la décision à prendre se résume à une question relativement simple:

- le corps médical donnera-t-il son aval à un ouvrage tarifaire sans aucun doute incomplet et qui devra encore être amélioré et ménagera-t-il ses forces pour des développements et des débats plus importants, 
ou bien

- le combat pour un ouvrage tarifaire harmonieux est-il si important pour lui, qu'il est prêt sacrifier son argent et ses positions stratégiques?

C'est sur cette question essentielle que porte la votation générale; celle-ci est donc totalement ouverte et n'a pas fait l'objet d'un mot d'ordre du Comité central à la Chambre médicale en faveur du OUI.

Dans cette situation très difficile, trois réactions sont visibles chez un grand nombre de membres:

1. Nous facturons selon notre propre tarif, par exemple selon un tarif de la FMS

Cette idée est tout simplement irréalisable:

- En Suisse, les systèmes tarifaires doivent être négociés avec les assureurs et recevoir l'approbation des autorités étatiques compétentes. Les actes unilatéraux sont par principe impensables du point de vue juridique (ce qui ne signifie pas que la FMH ne puisse pas élaborer correctement une structure tarifaire TARMED mettant en évidence les erreurs de la structure actuelle, ni qu'elle ne le fera pas).

- Ce principe général est illustré par le fait qu'en Suisse, conformément à l'art. 43, 5e al., LAMal déjà cité, une seule structure tarifaire doit être utilisée pour la facturation des prestations particulières.

2. Repousser la décision dans l'attente d'une meilleure structure tarifaire et tant que toutes les VPT et la date d'introduction ne sont pas connues Les divers organes ont reconnu que cette stratégie, si elle devait être adoptée, n'aurait aucun résultat:

- La décision doit être prise maintenant; tout renvoi sera interprété comme un non.

- Des améliorations de la structure tarifaire sont possibles, avant tout dans le domaine du modèle de facturation, et elles seront acceptées par les autres parties (cf. point 7). Mais elles ne pourront être réalisées que si la FMH accepte désormais le résultat actuel des négociations et reste ainsi à la table des négociations.

- Et répétons-le: l'espoir de pouvoir continuer à utiliser les anciens tarifs et de conserver l'ancien régime est illusoire car non conforme à la loi.
3. TARMED (n')est (plus) notre affaire; que «Madame Dreifuss» - personnifiant tout le monde politique - introduise le tarif TARMED et en assume ainsi l'entière responsabilité Cette position est aussi trompeuse que suicidaire:

- A court terme, elle peut permettre de gagner du temps et de se décharger.

- Mais elle ne pourra jamais empêcher l'introduction du TARMED ou d'un système de facturation qui en serait dérivé ... ni la fixation d'une valeur du point à la mode Dreifuss.

- Et, conséquence fatale: cette façon d'opérer constituerait un aveu de la part de la FMH que le corps médical n'est plus en mesure de gérer lui-même les affaires les plus fondamentales et que l'autonomie de la profession médicale et son organisation professionnelle appartiennent au passé. En d'autres termes, ce serait la fin de la Fédération des médecins suisses.

Celui qui, par faiblesse, invite les baillis dans son propre pays ne doit pas s'étonner si ceux-ci décident de s'y installer durablement. Cette vérité qui ressort des 700 ans d'expérience tant positive que négative de notre histoire conserve encore aujourd'hui toute sa validité d'un bout à l'autre de la Suisse.

Le Comité central et la Chambre médicale vous recommandent de voter trois fois OUI et d'approuver ainsi la structure tarifaire et les conventions-cadres pour le domaine AA/AM/AI et celui de la LAMal. 